\title{
Bilateral discoid medial menisci: a rare phenomenon
}

\author{
Puspak Samal ${ }^{1}$, Ms, Kishan Bhagwat ${ }^{1}$, MS, Tapas $\underline{\text { Panigrahi }}^{2}$, Ms, Nirmalraj Gopinathan ${ }^{1}$, MS
}

\begin{abstract}
Discoid medial meniscus is a relatively rare pathology of the knee joint, with bilateral cases even rarer. Herein, we report the case of a 25-year-old man diagnosed with discoid medial meniscus in the right knee with a horizontal tear. Increased cupping of the medial condyle of the tibia, widening of the medial joint space and the presence of discoid meniscus in the right knee prompted investigation of the asymptomatic left knee with magnetic resonance imaging. The contralateral asymptomatic knee also showed evidence of discoid medial meniscus. The symptomatic knee was successfully treated by arthroscopic partial meniscectomy, with excellent functional outcome.
\end{abstract}

Keywords: arthroscopy, discoid medial meniscus, knee

\section{INTRODUCTION}

Discoid meniscus is an uncommon cause of internal derangement of the knee. The discoid-shaped meniscus mostly occurs on the lateral side of the knee joint. This anomaly very rarely occurs in the medial side, with the first of such a case reported by Cave and Staples in 1941.(1) Discoid medial menisci in both knees (i.e. bilateral) is also rare. ${ }^{(2)}$ Since Murdoch reported the first case of bilateral discoid medial menisci in 1956, ${ }^{(3)}$ fewer than 20 cases have been reported in the literature. ${ }^{(4)}$ We herein report a case of bilateral discoid medial menisci with a tear on one side, which was successfully treated by arthroscopy.

\section{CASE REPORT}

A 25-year-old man presented with complaints of pain, recurrent swelling and occasional clicking of the right knee. He had an episode of a twisting injury to the knee while running on uneven surface one year earlier. The pain increased with walking for long distances, climbing stairs and squatting. It was also associated with recurrent episodes of knee joint effusion. Upon physical examination, quadriceps wasting and evidence of mild knee joint effusion were noted. Joint line tenderness and McMurray's tests for medial meniscus injury were positive. The range of motion (ROM) was $0^{\circ}-110^{\circ}$ of flexion and terminal movements elicited pain in the patient's right knee. There were no signs of instability in the knee joint, and the contralateral left knee was asymptomatic.

Standing anteroposterior and lateral radiography showed a widening of the medial joint space in both knees (Figs. 1 \& 2) and increased cupping of the medial condyle of the tibia in the right knee. Magnetic resonance (MR) imaging of the right knee revealed the classic 'bow tie' appearance in three consecutive sagittal images and a continuity of meniscal material approaching the intercondylar area. There was a horizontal tear of the posterior horn of the meniscus. Based on these findings, the diagnosis of discoid medial meniscus with a horizontal tear was made (Fig. 3). The lateral meniscus was normal. Although the left knee was asymptomatic, we assessed it to exclude bilateralism. MR imaging revealed the presence of a variety of discoid medial
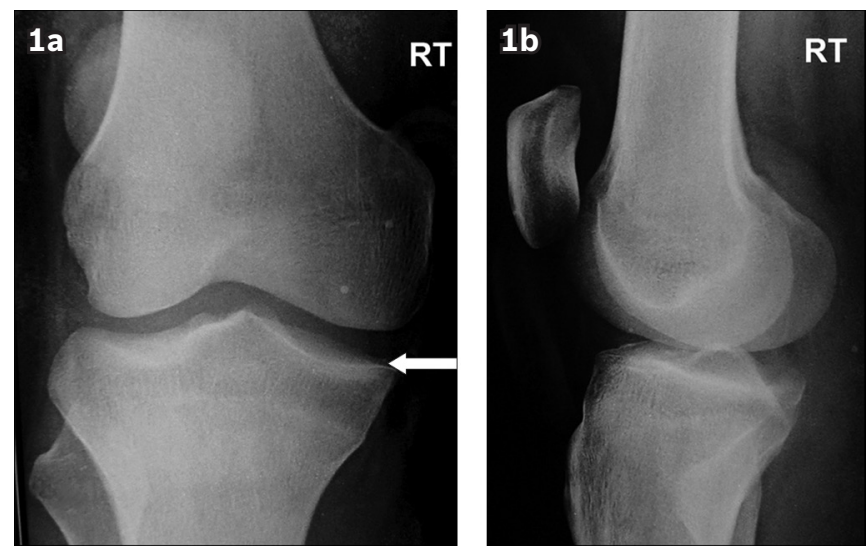

Fig. 1 (a) Anteroposterior and (b) lateral radiographs of the right knee show the widened medial joint and cupping of the medial condyle tibial plateau (arrow).
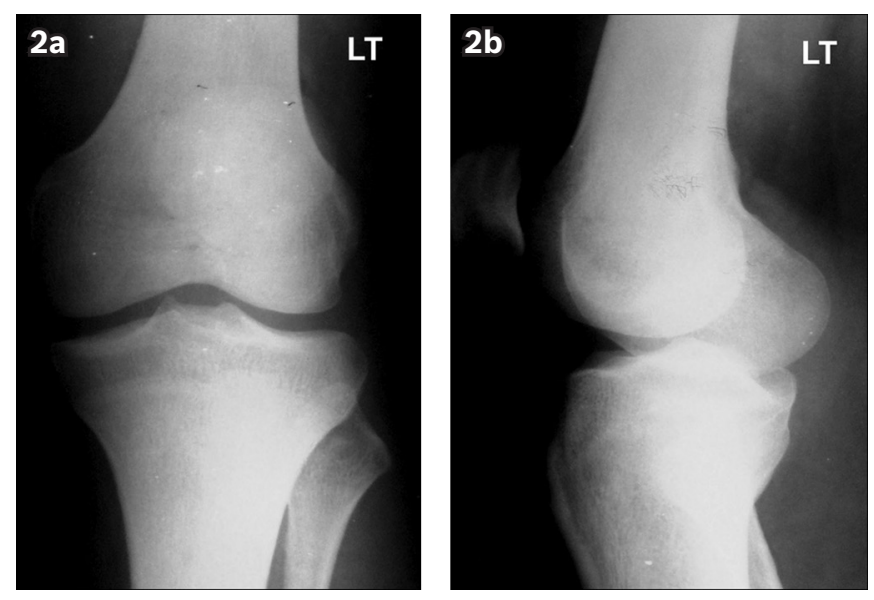

Fig. 2 (a) Anteroposterior and (b) lateral radiographs of the left knee show widening of the medial joint space.

meniscus with increased cupping of the medial condyle of the tibia (Fig. 4).

Arthroscopy of the symptomatic right knee confirmed the presence of discoid medial meniscus extending almost into the intercondylar area (Fig. 5a). Evidence of fatty degeneration was also seen around the intercondylar area and the posterior horn of the medial meniscus (Fig. 5b). The horizontal tear of the

${ }^{1}$ Department of Orthopaedics, Post Graduate Institute of Medical Education and Research, Chandigarh, ${ }^{2}$ Deparment of Orthopaedics, Shri Ramachandra Bhanj Medical College, Cuttack, Odisha, India

Correspondence: DrPuspak Samal, Registrar, Department of Orthopaedics, Post Graduate Institute of Medical Education and Research, Chandigarh, India. drpuspak27@gmail.com 

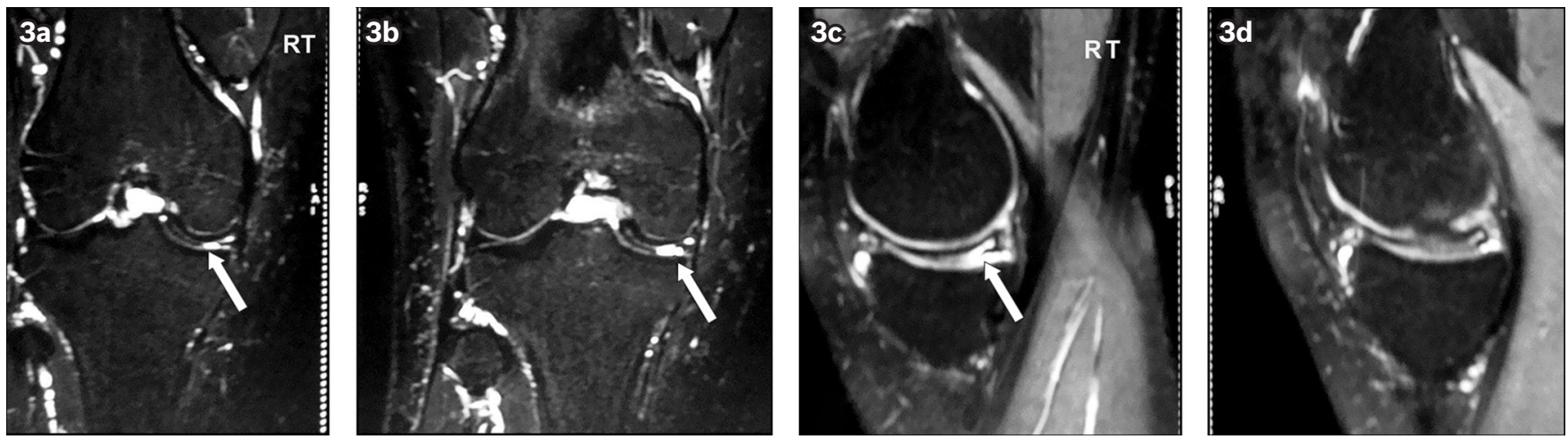

Fig. 3 ( $\mathrm{a} \& \mathrm{~b}$ ) Coronal MR images of the right knee show complete variety of discoid medial meniscus (arrows). (c \& d) Sagittal MR images of the right knee show horizontal tear of the posterior horn (arrow).

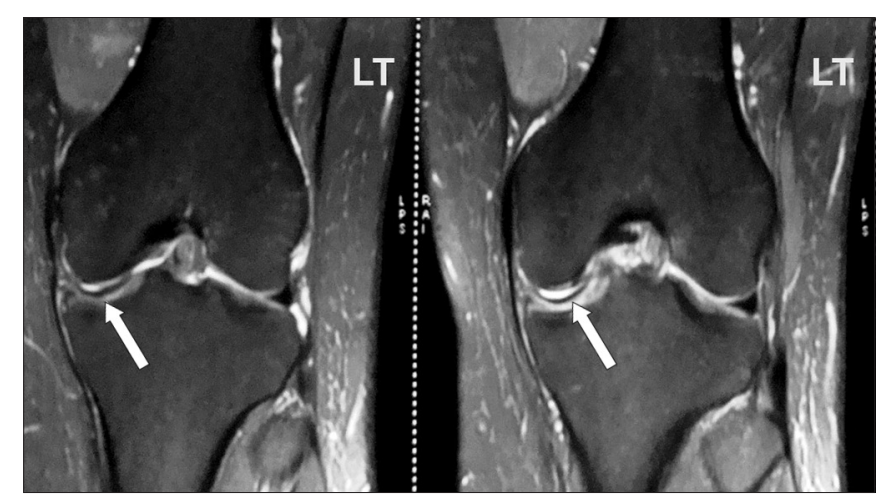

Fig. 4 Coronal MR images of the left knee show a complete discoid medial meniscus (arrows).
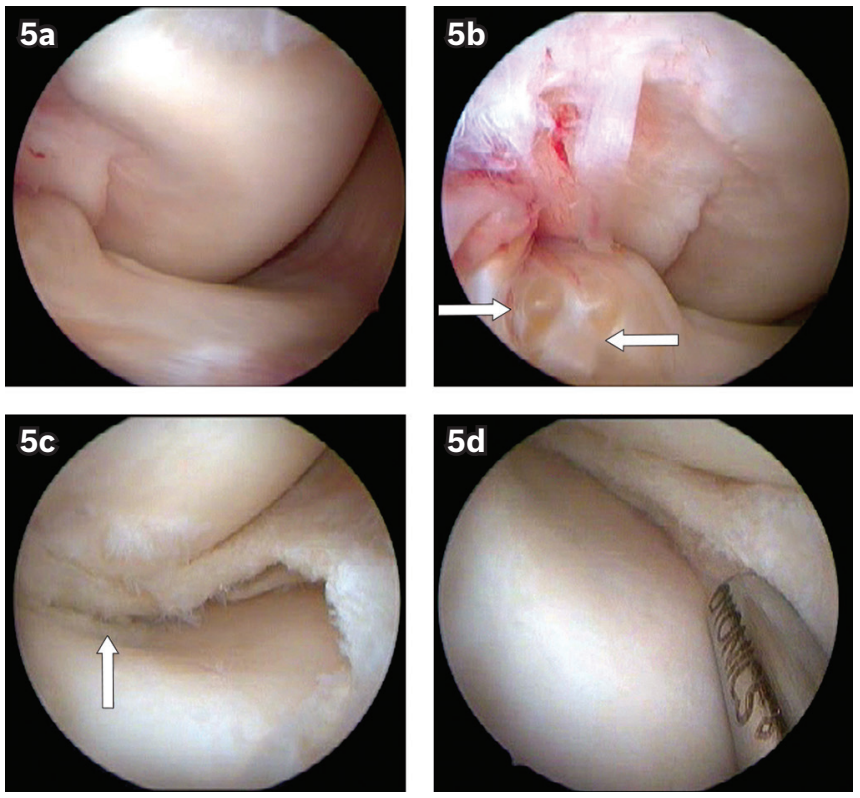

Fig. 5 Arthroscopic images of the right knee show (a) discoid medial meniscus extending to the intercondylar region, (b) fatty degeneration in the intercondylar region (arrows); (c) horizontal tear of the posterior horn (arrow); and (d) the medial meniscus after partial meniscectomy.

posterior horn extended from the midsubstance (Fig. 5c). Other intra-articular structures were normal. Judicious partial resection of the torn part of the medial meniscus was performed (Fig. 5d). The patient was allowed full weight-bearing and knee movement from the first postoperative day. At follow-up two years later, the patient was doing well with complete painless ROM.

\section{DISCUSSION}

Since the report by Cave and Staples in 1941, ${ }^{(1)}$ discoid medial meniscus still remains an uncommon clinical entity and bilateralism is exceedingly rare. The actual incidence of discoid medial meniscus and its bilateralism is difficult to cite because most patients are asymptomatic. Out of the 10,000 meniscectomies reported by Smillie, ${ }^{(5)} 467$ were done for discoid lateral meniscus and 7 for discoid medial meniscus. In 14,731 meniscal injuries examined by Dikason et al, ${ }^{\left({ }^{6}\right)} 10$ out of $8,040(0.12 \%)$ medial meniscal and 102 out of $6,691(1.52 \%)$ lateral meniscal injuries were discoid. The reported incidence of discoid medial meniscus was $0.06 \%-0.30 \%$ in various studies. ${ }^{(4)}$

The aetiology of discoid meniscus is controversial. In 1948, Smillie postulated that the discoid shape is normal in the developing embryo, ${ }^{(4,5)}$ and that failure of absorption of the central portion, which persists during the fetal stage, leads to 'congenital discoid meniscus'. (5) In 1974, Weiner and Rosenberg attributed discoid meniscus to congenital changes in the tibia. ${ }^{(7)}$ Stable discoid is often an incidental finding in asymptomatic patients. It can, however, be symptomatic in the presence of a tear, and the most common tear pattern is that of a degenerative horizontal cleavage, comprising $58 \%-98 \%$ of all symptomatic discoid menisci. ${ }^{(8,9)}$ Our patient had a large horizontal tear extending from the midsubstance to the posterior horn of the medial meniscus.

The radiological findings associated with discoid medial meniscus are: (a) cupping of the medial tibial plateau; (b) proximal tibial physis collapse; and (c) widening of the medial joint space. ${ }^{(2)}$ Atay et al reported a case where bilateral cupping of the medial tibial plateau was observed. ${ }^{(10)}$ In our case, there was bilateral widening of the medial joint space and an increased cupping of the medial condyles of the tibia.

MR imaging has facilitated the diagnosis of discoid meniscus. The presence of the 'bow tie' sign in more than three slices of sagittal plane MR images suggests the occurrence of discoid meniscus. ${ }^{(11)}$ In coronal plane MR imaging, the most accurate criterion for the diagnosis of discoid meniscus is when the ratio of the minimal meniscal width to the maximal tibial width is more than $20 \% .{ }^{(8)}$ However, arthroscopy remains the gold standard for diagnosing discoid meniscus. According to the Watanabe classification, ${ }^{(12)}$ our case had a complete variety of 
discoid medial meniscus on the right knee. Fatty degeneration around the posterior horn of the medial meniscus, as seen in our patient, was also observed by Nam in his case report. ${ }^{(2)}$ There are several types of anomalies associated with discoid medial meniscus, such as hypoplasia of the anterior horn, anomalous attachment of the posterior horn to the femoral condyle, and anomalous attachment to the anterior cruciate ligament; ${ }^{(11,13)}$ in our case, the rest of the intra-articular structures were normal.

Patel et $\mathrm{al}^{(14)}$ recommend that the discoid meniscus be preserved if severe symptoms are absent and advocate partial resection (i.e. saucerisation) of a torn symptomatic meniscus. In our case, we performed a partial meniscectomy until a stable peripheral rim was achieved. The patient was doing well at the two-year follow up. In general, the results of partial meniscectomy in discoid meniscus are good. However, only symptomatic cases should be treated surgically, as most cases of discoid meniscus remain asymptomatic. ${ }^{(15)}$

In conclusion, discoid medial meniscus is a rare abnormality. Good quality radiography and MR imaging aid in preoperative diagnosis. MR imaging-assisted preoperative planning can provide surgeons with the opportunity to plan for partial resection so as to lessen the chance of early onset of osteoarthritis. Bilateralism should be ruled out in every case of discoid medial meniscus so that the patient can take precautions to avoid a similar injury in the contralateral asymptomatic limb in the future.

\section{REFERENCES}

1. Cave EF, Staples OS. Congenital discoid meniscus: a cause of internal derangement of the knee. Am J Surg 1941; 54:371-6.

2. Nam TS. Bilateral discoid medial menisci: Two different types in one patient and bony changes on the medial tibial plateau. J Korean Orthop Assoc 2011; 46:172-6.

3. Murdoch G. Congenital discoid medial semilunar cartilage. J Bone Joint Surg Br 1956; 38-B:564-6.

4. Lee JH, Wang SI, Park JH, Lim YJ. A case of asymmetric bilateral discoid medial menisci. J Korean Knee Soc 2011; 23:55-9.

5. Smillie IS. The congenital discoid meniscus. J Bone Joint Surg Br 1948; 30B:671-82.

6. Dickason JM, Del Pizzo W, Blazina ME, et al. A series of ten discoid medial menisci. Clin Orthop Relat Res 1982; (168):75-9.

7. Weiner B, Rosenberg N. Discoid medial meniscus: association with bone changes in the tibia. A case report. J Bone Joint Surg Am 1974; 56:171-3.

8. Yaniv M, Blumberg N. The discoid meniscus. J Child Orthop 2007; 1:89-96.

9. Kim SJ, Lubis AM. Medial and lateral discoid menisci: a case report. Sports Med Arthrosc Rehabil Ther Technol 2010; 2:21.

10. Atay OA, Doral MN, Aydingoz U, Leblebicioglu G. Bilateral discoid medial menisci: association with bone changes in the tibia. Knee Surg Sports Traumatol Arthrosc 2001; 9:217-20.

11. Gad MS, Ezzat F, Nass MA. Discoid Medial Meniscus (Case Report). Pan Arab J Orth Trauma 2007; 11:118-21.

12. Watanabe M, Tekeda S, Ikeuchi H. Atlas of Arthroscopy. 3rd ed. Berlin: Springer-Verlag, 1979.

13. Tibrewal S, Garg S, Tibrewal SB. A symptomatic anamolous variant of the medial meniscus. A case report and review of the literature. J Orthopaedics 2007; 4:E28.

14. Patel D, Dimakopoulos P, Denoncourt P. Bucket handle tear of a discoid medial meniscus. Arthroscopic diagnosis--partial excision. A case report. Orthopedics 1986; 9:607-8.

15. Tachibana $Y$, Yamazaki Y, Ninomiya S. Discoid medial meniscus. Arthroscopy 2003; 19:E12-8. 